\section{Indirect laryngoscopy}

I enjoyed the CMAJ article by Trottier and colleagues, ${ }^{1}$ and wish to suggest a possible addition to the initial management of hoarseness algorithm in Figure 3.

Many primary care and emergency physicians are able to perform simple, indirect laryngoscopy with topical anesthesia, a headlamp and a laryngeal mirror. In a cooperative patient, obtaining a decent view of the glottic opening and surrounding area is often possible, and might allow physicians to decide quickly if a patient needs to see an otolaryngologist on an urgent basis. If the physician is unable to obtain a useful view, the algorithm continues as already described. ${ }^{1}$

Although many emergency physicians have access to and are trained in the use of a fiberoptic nasopharyngoscope, I think the indirect examination may still have a role in certain settings. We should endeavor to pass this skill on to trainees we mentor.

\section{Greg J. Korzan MD}

Department of Emergency Medicine, Kelowna General Hospital, Kelowna, BC

\section{Reference}

1. Trottier AM, Massoud E, Brown T. A case of hoarseness and vocal cord immobility. CMAJ 2013; 185:1520-4.

CMAJ 2014. DOI:10.1503/cmaj.114-0018

\section{Advocating for equality for preterm infants}

This letter relates to a $C M A J$ commentary by Batton and Batton. ${ }^{1}$ As parents of a preterm child, the term "significant physical and developmental issues" represented a grey area for us.

In our extensive readings, we came across many nebulous terms referring to outcome: "language impairments with lasting effects," "cognitive-developmental disorders," "dysfunction," "unfavourable outcome," "functional and socialbehavioural deficits" and "mental and motor impairments." There were many other examples. The National Institute of Child Health and Human Development preemie outcome calculator gave our daughter a $47 \%$ chance of dying or having "moderate to severe neurodevelopmental impairment." The greatest challenge for us was understanding the meanings of these terms. We knew that severe impairments included deafness, blindness, cerebral palsy and profoundly low IQ, but what about a "moderate" impairment? What is "dysfunction"? The only weakness in our interactions with the doctors caring for our baby was in understanding the meaning of the term "significant physical and developmental issues." We did not necessarily need numbers, odds or statistics, but rather, real-life examples. A more open, clear dialogue between doctors and parents is imperative.

\section{Rebecca L. Pearce}

\section{Reference}

1. Batton D, Batton B. Advocating for equality for preterm infants. CMAJ 2013;185:1559-60.

CMAJ 2014. DOI:10.1503/cmaj.114-0019

\section{Autism spectrum disorder: importance of audiology}

As Director of Audiology for the Canadian Association of Speech-Language Pathologists and Audiologists (CASLPA), I read with great interest, the $C M A J$ article on autism spectrum disorder by Anagnostou and colleagues. ${ }^{1}$ Congratulations to the authors.

The CASLPA is a national professional association that represents more than 6000 speech-language pathologists, audiologists and supportive personnel - many of whom work closely, and daily, with patients or clients affected by autism spectrum disorder. Anagnostou and colleagues ${ }^{1}$ have done a commendable job of noting the role of speech-language pathologists and the importance of comprehensive assessment and intervention of communication disorders. However, the importance of a comprehensive audiologic assessment was overlooked and was particularly missed in the section that outlines best practice for clinical work-up for autism spectrum disorder. Box 4 in the article, ${ }^{1}$ which lists red flags for autism, includes indices for delayed or atypical language. Some of these red flags are also signs of possible hearing loss.

Although there are challenges involved in providing diagnoses for young children, advances in auditory physiologic testing permit audiologists to assess auditory function with accuracy in young children.

I understand that not all professions that may affect the diagnosis of autism spectrum disorder could be listed in this article; however, I strongly believe that audiologists play too valuable a role on the interprofessional team to be excluded. Determining auditory function is a fundamental, necessary step in diagnosing and providing interventions for autism spectrum disorder. I respectfully suggest that more reference to audiologic assessment be included as part of the best practice model for diagnosing autism spectrum disorder.

\section{Chantal Kealey AuD Aud(C)}

Director of Audiology, Canadian Association of Speech-Language Pathologists and Audiologists, Ottawa, Ont.

\section{Reference}

1. Anagnostou E, Zwaigenbaum L, Szatmari P, et al. Autism spectrum disorder: advances in evidencebased practice. CMAJ 2014; Jan. 13. [Epub ahead of print].

CMAJ 2014. DOI:10.1503/cmaj.114-0020

\section{CORRECTION}

\section{Error in table heading}

In the article "Prevalence of and factors associated with head impact during falls in older adults in long-term care," column 4 of Table 2 should read "Probability of head impact (95\% CI)" rather than "OR for head impact (95\% CI)." CMAJ apologizes for this error.

\section{Reference}

1. Schonnop R, Yang Y, Feldman F, et al Prevalence of and factors associated with head impact during falls in older adults in long-term care. CMAJ 2013; 185:E803-10.

CMAJ 2014. DOI:10.1503/cmaj.114-0021 